\title{
Ultrastructure of the Erythroblasts in Mice Splenic Colonies
}

\author{
Akira B. Mrura, Kosaku Yoshida, Akiniko Yamaguchi \\ and Mitsuyuki Fukuda \\ The Third Department of Internal Medicine, Akita University \\ School of Medicine, Akita 010
}

\begin{abstract}
Mrura, A.B., Yoshida, K., Yamaguchi, A. and FUKUda, M. Ultrastructure of the Erythroblasts in Mice Splenic Colonies. Tohoku J. exp. Med., 1978, 125 (4), 353-365 — Ultrastructure of the erythroblasts in mice splenic colonies was studied in contradistinction to that found in the normal mice spleen and bone marrow. Although the basic morphology of erythroblasts in those tissues was identical, most of the erythroblasts in splenic colonies were in the early stage, and those in normal mice were in the intermediate or late stage. A very few or no microtubules were seen in the erythroblasts in normal spleen and colonies, while they were often recognized in the marrow erythroblasts. The micropinocytic vesicles were often seen in the marrow erythroblasts. There was no distinct structure delimiting the erythroblasts colonies from the surrounding tissue cells. Furthermore, macrophages were often recognized in the central part of colonies. Some macrophages engulfed and digested the erythroblasts. However, attachment apparatus such as desmosome, or the delivery of iron particles from macrophages to erythroblasts was never observed.__ erythroblasts; ultrastructure; mice splenic colonies
\end{abstract}

Each of the splenic colonies of the lethally irradiated mice to which the bone marrow cells were transfused after irradiation is presumed to derive from a single multipotential stem cell. A few days after marrow transplantation, a certain extent of differentiation takes place and a large number of early and intermediate erythroblasts are present in the splenic colonies. Although the way of the cell differentiation is of special interest, there are few reports available concerning the morphological features of colony forming hematopoietic cells.

It is the purpose of this paper to describe the ultrastructural development of the erythroblasts in the splenic colonies.

\section{Materials and Methods}

8 to 12 -week-old C57BL mice were used in the experiments. Lethally irradiated mice were injected with $1 \times 10^{5}$ normal bone marrow cells taken from the same strain of mouse according to the original method by Till and McCulloch (1961).

Two irradiated recipient mice were killed each on the seventh, ninth, eleventh and fourteenth days. The spleens of these mice were fixed in $2.5 \%$ glutaraldehyde with subsequent fixation in $1 \%$ osmium tetroxide, dehydrated in a graded series of ethanol, and embedded in Epon mixture. The ultrathin sections were stained with uranyl acetate

Received for publication, January 12, 1978. 
and lead, and examined in a JEM $100 \mathrm{~B}$ or $100 \mathrm{C}$ type electron microscope.

In order to compare with the colony erythroblasts, the bone marrow and the spleen of the mice without any treatment were also studied.

\section{RESULTS}

Normal morphology of developing erythroblasts in the bone marrow and the spleen

Most of the erythroblasts in the bone marrow and spleen were in the intermediate or the late stage (Figs. 1, 2, 3, 4, and 5). The primitive or early erythroblasts were difficult to find out. The fine structure of the erythroblasts has been described by some authors (Bessis 1967; Miura et al. 1974), and results of this study agree to these descriptions on the whole.

A great number of erythroblasts in normal mice had a condensed nucleus with a little euchromatinic space. No prominent nucleoli were observed in it. The cytoplasm of the intermediate erythroblasts contained numerous free ribosomes, a few mitochondria and a small Golgi zone. Occasionally the centrioles and siderosomes were observed. With further maturation, fewer ribosomes were visualized and the cytoplasm contained homogeneous materials identified as hemoglobin.

There were two great differences between the marrow and the spleen erythroblasts. In the erythroblasts of the marrow, a good number of micropinocytoses and coated vesicles were observed (Fig. 3), and a few short microtubules run in the cytoplasm (Figs. 4 and 5). In contrast, there were a few coated vesicles but no microtubules in the splenic erythroblasts (Fig. 2).

Ultrastructure of the erythroblasts in the splenic colonies

On the seventh day after marrow transplantation, nodules in the spleen were not so distinct as to be discriminated macroscopically. However, ultrastructural observations revealed colonies of rapidly proliferating erythroblasts.

On the ninth day, the splenic colonies became obvious macroscopically and they became soft and crumbly on the fourteenth day.

Almost all erythroblasts in the seventh or ninth day were in the early stage. The late erythroblasts were sometimes observed on the fourteenth day.

The size of erythroblasts decreased with maturation from about 12 to $5 \mu \mathrm{m}$ in diameter.

The nucleus of the primitive erythroblasts was large and irregular in shape (Fig. 6). It contained a prominent nucleolus. The nucleoplasm was mostly composed of euchromatin. Cytoplasm was filled with a large number of free ribosomes. A few large mitochondria, often partly ruptured, were observed in each erythroblast. Golgi apparatus, centrioles and siderosomes were sometimes recognized (Fig. 7).

Differentiation in the erythroblasts involved progressive reduction in the nuclear and cytoplasmic sizes and condensation of nuclear chromatin (Fig. 8). 
The nucleus became increasingly eccentric in position until finally it was attached to the rest of the cell by only a narrow cytoplasmic bridge. Free ribosomes decreased in number in proportion to the increase of matrix density. Mitochondria in the late erythroblasts were usually small and preserved the inner structure well.

A few micropinocytic vesicles and coated vesicles were observed. On the other hand, no microtubules were recognized with few exceptions.

There were no capsules or fibrous structures delimiting between erythroblasts and surrounding tissue cells. In the periphery of the colonies, erythroblasts intermingled with fibroblasts, lymphocytes, plasma cells and macrophages. No attachment structures such as desmosomes or tight junctions were observed between erythroblasts and other kinds of cells or among erythroblasts.

The macrophages were often recognized not only around but within the erythroblasts colonies (Fig. 9). Occasionally, the erythroblasts were engulfed and digested by the macrophages (Fig. 10). There was no evidence that the erythroblasts directly took in ferritin particles from the macrophages.

\section{Discussion}

Epoch-making progress has been made in the studies of hematopoietic stem cells by Till and McCulloch (1961) who successfully conducted the experiment in which the lethally irradiated mice were transplanted with bone marrow. Their study demonstrated that erythroblasts and granulocytes as well as megakaryocytes that appear in splenic colonies differentiate from a single stem cell. Although no such definite experimental evidence has been achieved in man, yet from some clinical facts the occurrence of hematopoietic stem cells is commonly accepted.

Splenic colonies that proliferate from the transplanted stem cells consist mostly of erythroblasts at first. Granulocytes and megakaryocytes increase with time. The calculation of splenic colonies in mice is, in general, made 7-9 days after transplantation. However, there are few papers describing systematically the ultrastructures of cells in splenic colonies. The present study was intended to describe the morphology of erythroblasts in such colonies. Since early erythroblasts usually make up the major part of the colonies not more than 9 days after transplantation, which are used in common experiments, our observation was made of splenic colonies not more than 14 days after transplantation.

The morphology of stem cells themselves is not definitely known. Not a few electron micrographs have been presented as showing stem cells (Murphy et al. 1971; van Bekkum et al. 1971; Dicke et al. 1973; Fukuda 1973, 1974), but there is no definitive evidence to support them. Splenic colonies have likewise been studied, but the stem cells have not yet been identified, for it is difficult to label them with $\mathrm{H}^{3}$-thymidine because of poor metabolism at the stage of stem cells (Fliedner et al. 1964). Recently, the dominant view has been that the stem cells are considered to be small lymphoid, but the stem cells could not be identified in our observation, either.

Erythroblasts are normally observed in the bone marrow and spleen of 
C57BL mice. The basic structure of the cells at either site is similar to that found in the human erythroblasts and it is not difficult to identify them. Early erythroblasts are rare, and erythroblasts at the intermediate and late stages make up the majority of them. On the other hand, early erythroblasts comprise almost all the erythroblasts in the splenic colonies not more than 9 days after transplantation, and late erythroblasts do not increase until 14 days after transplantation. Even at this stage, early erythroblasts are still predominant; and although it is not easy to accurately compare them with erythroblasts in the bone marrow and spleen of normal mice, both cells are basically identical in the following aspects: The cells are reduced in size as they grow. Their nuclei are larger, the earlier the stage is, and they consist only of euchromatin and nucleoli, and are often irregular in shape. Heterochromatin gradually increases with maturation, and the nuclei gradually assume a regular round shape. Mitochondria are large, and likely to be destroyed at fixation at the early stage. In mature erythroblasts, however, they are small and retain their internal structure. These findings agree with our previous descriptions (Miura et al. 1974).

It is of interest to note that a very few or no microtubules are found in the erythroblasts in the normal spleen or splenic colonies, whereas microtubules are frequently found in the erythroblasts in the normal mouse bone marrow. In our previous observation of erythroblasts in the human bone marrow, we have also disclosed that microtubules are abundant and occur in various shapes (Miura et al. 1974).

On the other hand, there are no papers indicating the occurrence of microtubules in the erythroblasts in the spleen. Little is known about the significance of microtubules in erythroblasts. Only the role of the microtubules found in mitotic spindles and mid-bodies, as the rest of mitotic spindle, is all that we know. The potential role as what is called the cytoskeletal system as well as other roles may be considered. The fact that microtubules are difficult to find in the spleen but remarkable in the bone marrow is very suggestive, but its functional significance still remains to be studied.

Generally, there are very few siderosomes in the erythroblasts in splenic colonies. Micropinocytic veiscles are often found while the occurrence of iron particles in the vesicles is infrequent. These findings agree with the reports of Berman (1967) in mice erythroblasts.

Because a number of erythroblasts grow synchronously in the splenic colonies, it may be useful to study the change of ultrastructure under different experimental conditions.

\section{References}

1) Berman, I. (1967) The ultrastructure of erythroblastic islands and reticular cells in mouse bone marrow. J. Ultrastruct. Res., 17, 291-313.

2) Bessis, M. (1967) Morphology of the different stages of maturation of the cells of the erythrocytic series in mammals. Exp. Biol. Med., 1, 220-233.

3) Dicke, K.A., van Noord, M.J., Maat, B., Schaefer, U.W. \& van Bekkum, D.W. (1973) 
Identification of cells in primate bone marrow resembling the hemopoietic stem cell in mouse. Blood, 38, 547-558.

4) Fliedener, T.M., Thomas, E.D., Meyer, L.M. \& Cronkite, E.P. (1964) The fate of transfused [ $\left.{ }^{3} \mathbf{H}\right]$ thymidine-labeled bone marrow cells in irradiated recipients. Arn. N.Y. Acad. Sci., 114, 510-527.

5) Fukuda, T. (1973) Fetal hemopoiesis. I. Electron microscopic studies on human yolk sac hemopoiesis. Virchows Arch. Abt. B. Zellpath., 14, 197-213.

6) Fukuda, T. (1974) Fetal hemopoiesis. II. Electron microscopic studies on human hepatic hemopoiesis. Virchows Arch Abt. B. Cell Path., 16, 249-270.

7) Miura, A.B., Shibata, A., Akihama, T., Endo, Y. \& Saito, Y. (1974) Ultrastructure of the developing erythrocytes. Tohoku J. exp. Med., 112, 299-313.

8) Murphy, M.J., Bertles, J.F. \& Gordon, A.S. (1971) Identifying characteristics of the haematopoietic precursor cell. J. Cell Sci., 9, 23-47.

9) Till, J.E. \& McCulloch, E.A. (1961) A direct measurement of the radiation sensitivity of normal mouse bone marrow cells. Rad. Res., 14, 213-222.

10) van Bekkum, D.W., van Noord, M.J., Maat, B. \& Dicke, K.A. (1971) Attempts at identification of hemopoietic stem cell in mouse. Blood, 38, 547-558. 
Fig. 1. Erythroblasts in normal mouse bone marrow. A cytoplasmic bridge called a midbody connects two intermediate erythroblasts. Microtubules are seen within midbody. $\times 8,400$.

Fig. 2. Early and intermediate erythroblasts in normal mouse spleen. The cytoplasm is filled with free ribosomes. $\times 11,700$. 


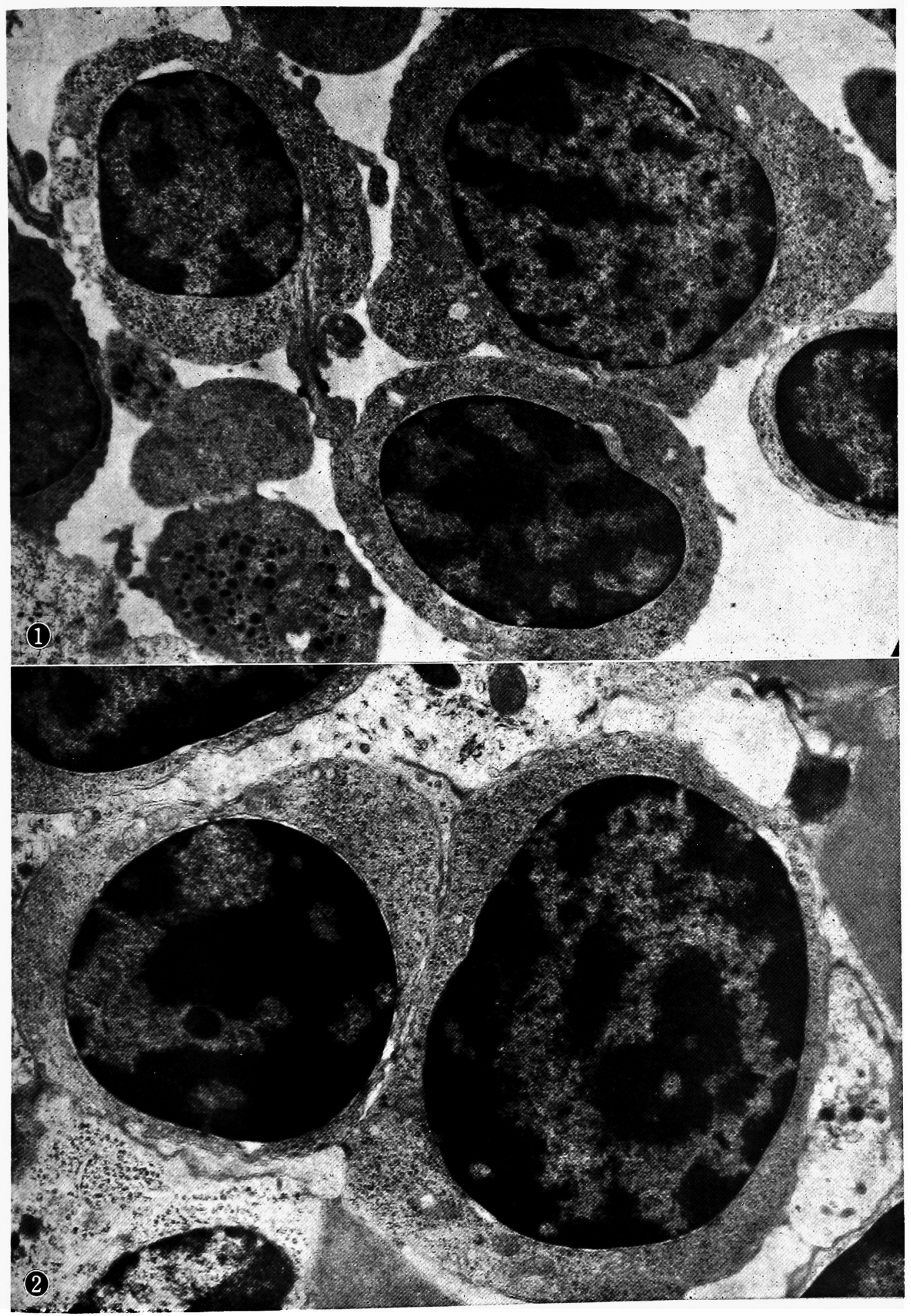


Fig. 3. Late erythroblast and naked nucleus in bone marrow. The free ribosomes decrease in number in inverse proportion to the increase of amorphous materials regarded as hemoglobin. $\times 11,500$.

Figs. 4 and 5. Microtubules in the marrow erythroblasts. In Fig. 4, they are located close to the nucleus. $\times 24,000 . \times 26,000$. 

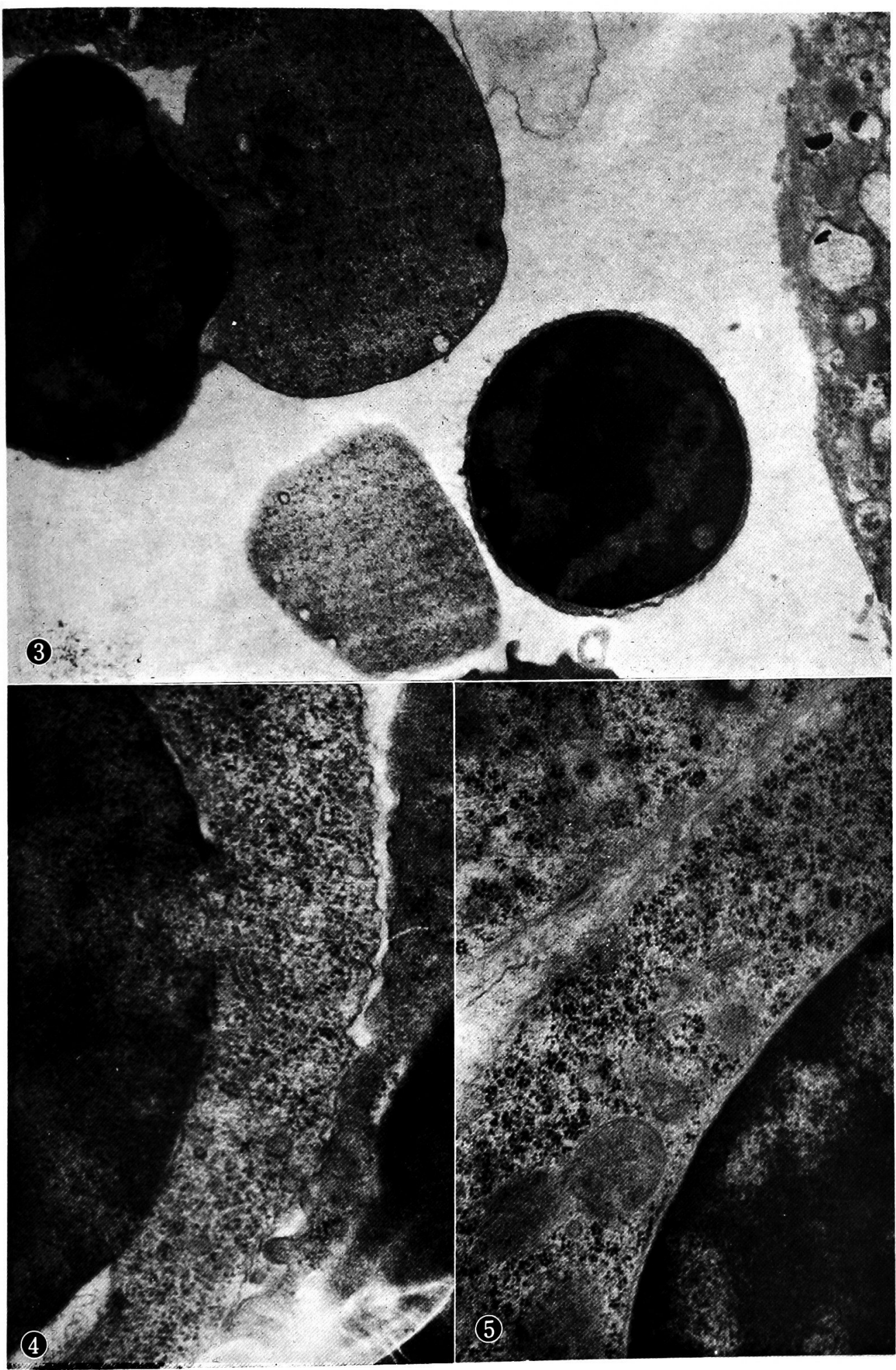
Fig. 6. Early erythroblast in the 7th day splenic colony. The large irregular shaped nucleus is mostly composed of euchromatin with the exception of a prominent nucleolus. The cytoplasm is filled with free ribosomes. A few mitochondria appeared to be swollen and ruptured. $\times 9,000$.

Fig. 7. The Golgi area of early erythroblast in the 7 th day splenic colony. $\times 22,000$. 


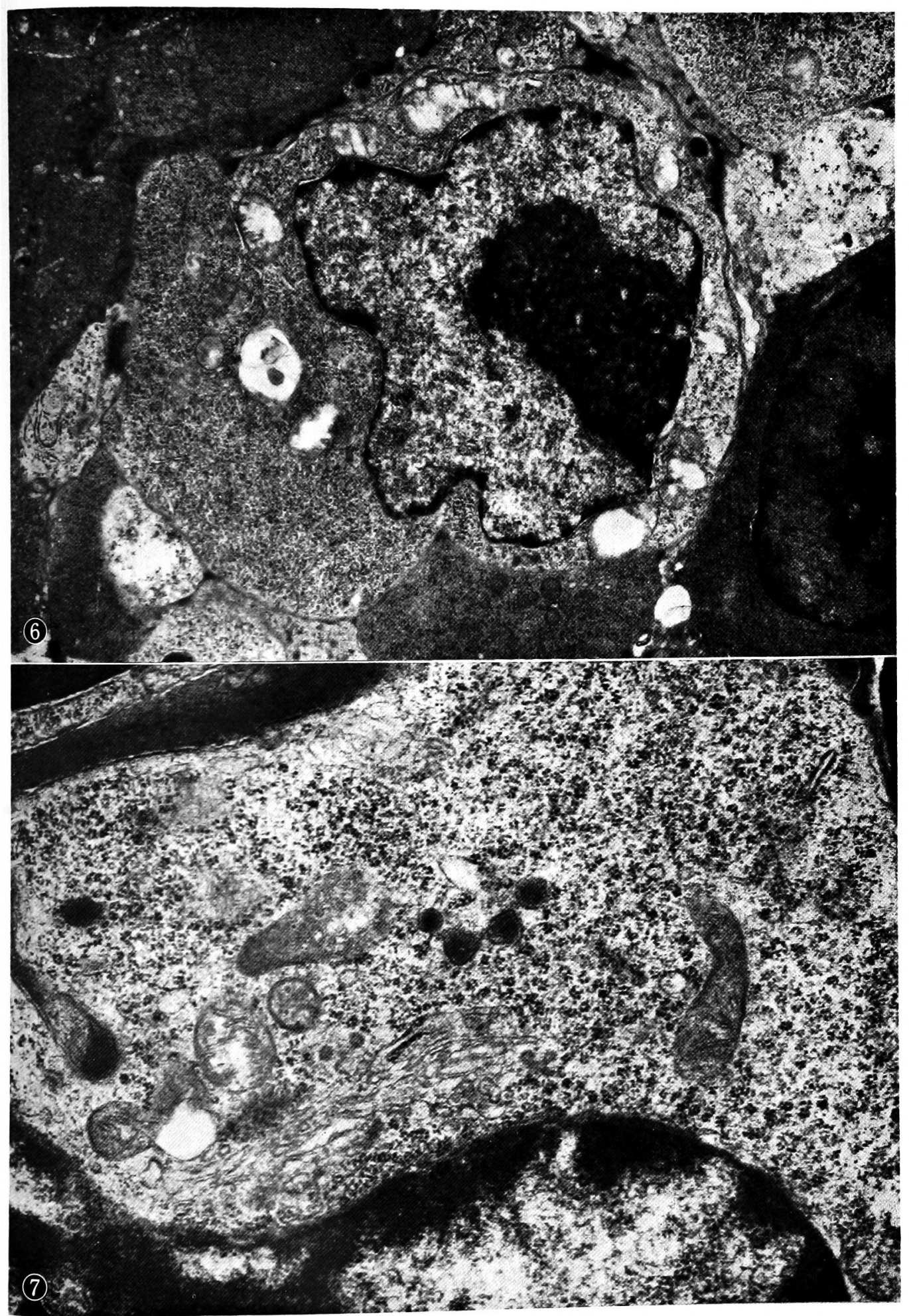


Fig. 8. Late erythroblast in the 14th day splenic colony. A few micropinocytic vesicles are recognized. $\times 15,000$.

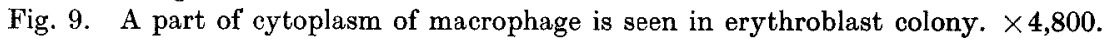

Fig. 10. The erythroblast phagocytized by a macrophage in the splenic colony. $\times 6,000$. 




